\title{
Islands of speciation or mirages in the desert? Examining the role of restricted recombination in maintaining species
}

\author{
MAF Noor and SM Bennett \\ Biology Department, Duke University, Durham, NC, USA
}

\begin{abstract}
Over the past decade, many studies documented high genetic divergence between closely related species in genomic regions experiencing restricted recombination in hybrids, such as within chromosomal rearrangements or areas adjacent to centromeres. Such regions have been called 'islands of speciation' because of their presumed role in maintaining the integrity of species despite gene flow elsewhere in the genome. Here, we review alternative explanations for such patterns. Segregation of ancestral
\end{abstract}

variation or artifacts of nucleotide diversity within species can readily lead to higher $F_{S T}$ in regions of restricted recombination than other parts of the genome, even in the complete absence of interspecies gene flow, and thereby cause investigators to erroneously conclude that islands of speciation exist. We conclude by discussing strengths and weaknesses of various means for testing the role of restricted recombination in maintaining species.

Heredity (2009) 103, 439-444; doi:10.1038/hdy.2009.151

Keywords: introgression; chromosomal rearrangements; recombination; speciation

A major focus of evolutionary genetic research has been to decipher causes of speciation from patterns of nucleotide polymorphism and divergence. In particular, researchers infer gene flow between related species and use these results to reject models of species formation wherein complete barriers to gene flow evolved during periods of geographic isolation (allopatry). Because 'the number of [recent] studies focusing on testing hybridization between species has increased by orders of magnitude' (Stevison, 2008), expressions such as 'speciation with gene flow' have become commonplace in the literature to describe cases of gene flow putatively occurring during initial species divergence and/or after secondary contact.

However, shared variation predating speciation ('lineage sorting') creates patterns often mistaken for gene flow between diverging species (see Hey, 2006 for review). To address this complication, several statistical models of DNA sequence evolution apply coalescent principles or other approaches to distinguish these possibilities (Wakeley and Hey, 1997; Machado et al., 2002; Hey and Nielsen, 2004; Becquet and Przeworski, 2007; Joly et al., 2009). Although these models are used extensively, known deviations from their assumptions in particular systems or inappropriate data sets (for example, microsatellite polymorphism rather than DNA sequence) cause investigators to resort to more basic predictions in testing for interspecies introgression.

Correspondence: Professor MAF Noor, Biology Department, Duke University, Box 90338, Durham, NC 27708 USA.

E-mail: noor@duke.edu

This paper is dedicated to Professor Jerry 'King' Coyne on the event of his 60th birthday.

Received 28 July 2009; revised 22 September 2009; accepted 25 September 2009
Perhaps the most common test for gene exchange is to determine whether some regions are significantly more differentiated between species than putatively 'neutral' regions or relative to the overall distribution of divergences observed. Although identified by divergence alone, such regions may bear alleles conferring adaptation or reproductive isolation between species. Relative divergence measures like $\mathrm{F}_{\mathrm{ST}}$ in particular have been advocated and used to test the importance of such regions in promoting adaptation or speciation in the face of gene flow (Beaumont, 2005).

One hypothesis that received particular attention in the past decade is that chromosomal rearrangements, or other regions of the genome in which recombination is rare or absent in species hybrids, are associated with creating or maintaining young species despite gene flow (Butlin, 2005; Hoffmann and Rieseberg, 2008). Theoretical models predict that regions of restricted recombination may facilitate species formation or persistence by creating linkage disequilibrium along large swaths of the genome including alleles conferring adaptation or barriers to gene flow (Noor et al., 2001c; Rieseberg, 2001; Navarro and Barton, 2003). Various lines of empirical data also support this idea: rearrangements are detected at lower genetic divergence in co-occurring species than in allopatric species (Noor et al., 2001c; Ayala and Coluzzi, 2005; Kandul et al., 2007), traits that prevent gene flow between species (such as habitat choice, mate preference or hybrid sterility) preferentially map to rearranged regions of the genome (Noor et al., 2001b; Feder et al., 2003), and most commonly, inverted regions tend to show greater nucleotide differentiation between species than regions not inverted (see below).

Here, we review several problems associated with using patterns of nucleotide differentiation (especially 
relative measures such as $\mathrm{F}_{\mathrm{ST}}$ or $\mathrm{Da}$ ) to test the role of restricted recombination in maintaining species. We discuss how restricted recombination can create regions of low intraspecific variation that, in comparison to regions of normal recombination, lead researchers to conclude differential gene flow among segments of the genome even if the species have never hybridized. The expression 'islands of speciation' (Turner et al., 2005) was coined to analogize genetic material being exchanged between species to flowing ocean water, but we conclude that the water (gene flow) itself may be a 'mirage' at times.

\section{Chromosomal rearrangements}

Studies of various taxa have shown higher divergence in rearranged than collinear regions between diverging species, including Drosophila species (Noor et al., 2007; Machado et al., 2007a, b), shrews (Basset et al., 2006, 2008; Yannic et al., 2009), Anopheles mosquito races (Michel et al., 2006) and Rhagoletis fruit flies (Feder et al., 2003). Early evidence also supported this model in Helianthus sunflowers (Rieseberg et al., 1999), though later studies suggested this effect may be localized to regions immediately adjacent to the rearrangement break points (Yatabe et al., 2007; Strasburg et al., 2009). However, support has not been universal-some species clearly hybridize extensively and persist without rearrangements (for example, Llopart et al., 2005), and some studies report regions of high differentiation widely distributed across the genome rather than clustered to specific rearrangements (see review in Nosil et al., 2009). Nonetheless, this prediction has been upheld in many systems tested and interpreted as evidence for a role of regions of restricted recombination in maintaining species despite ancient or recent hybridization.

However, rearranged regions may exhibit higher nucleotide divergence between species than collinear regions even if the species do not hybridize at all (Table 1). As such, this observation does not necessarily support a role of restricted recombination in allowing species to persist. First, multiple chromosomal rearrangements such as inversions segregate within many species (for example, Lewontin et al., 1981; Powell et al., 1999; Singh, 2001). Such inversions reduce recombination (and homogenization) from the time that they arise, particularly for short inversions and particularly near the inversion break points. If the different arrangements (for example, 'inverted' vs 'uninverted') persist within the species for some time and eventually alternately fix within subpopulations, the pattern of higher divergence in regions inverted between the species will appear. However, this higher divergence reflects the more ancient coalescence of the inverted regions relative to the collinear regions in the ancestor rather than 'speciation with gene flow.' Given the ubiquity of chromosomal rearrangements segregating within species, this pattern is likely to arise by chance and would result in inverted regions displaying greater nucleotide differentiation between species than regions not inverted, even in nonhybridizing species.

Second, chromosomal rearrangements have another biasing complication more directly associated with their recombination-reducing effect. Such rearrangements may often spread through directional selection (for example, Hoffmann and Rieseberg, 2008; Kirkpatrick and Barton, 2006). As with the spread of any adaptive variant, other sites will 'hitchhike,' and nucleotide diversity will be reduced near the selected site (MaynardSmith and Haigh, 1974). However, as a new chromosomal rearrangement spreads within a population, its spread will eliminate nucleotide diversity across a much wider swath of the genome because the entire segment (potentially megabases large) is linked as a single unit. The temporary reduction in nucleotide diversity within a subpopulation bearing the rearrangement will artifactually increase relative divergence measures such as $\mathrm{F}_{\mathrm{ST}}$ or $\mathrm{Da}$. These relative measures subtract or divide within-species diversity from total between-species divergence, so a reduction in the former will necessarily inflate the relative divergence measure irrespective of whether any interspecies gene flow has occurred.

\section{Centromeric regions}

Other recent studies have observed greater differentiation between diverging taxa near centromeres, potentially associated with their highly reduced recombination rates. This pattern has been documented repeatedly in Anopheles mosquito races (Stump et al., 2005; Turner et al., 2005; Slotman et al., 2006), but also in rabbits (Geraldes

Table 1 Biases that may lead to empirical observations mimicking a role for restricted recombination in maintaining species

\begin{tabular}{|c|c|c|}
\hline Empirical observation & Potential concern & Artifactual effect of potential concern \\
\hline $\begin{array}{l}\text { Higher nucleotide divergence } \\
\text { between species in rearranged } \\
\text { than collinear regions }\end{array}$ & $\begin{array}{l}\text { Rearrangement could have predated species split, } \\
\text { and divergence in rearranged region reflects the } \\
\text { time since it arose rather than species split }\end{array}$ & $\begin{array}{l}\text { Observe greater divergence in rearranged than } \\
\text { collinear regions }\end{array}$ \\
\hline As above & $\begin{array}{l}\text { Recent spread of arrangement by natural selection } \\
\text { reduced nucleotide diversity within one species }\end{array}$ & $\begin{array}{l}\text { Observe inflation in relative divergence measures } \\
\text { because diversity within species reduced }\end{array}$ \\
\hline $\begin{array}{l}\text { Higher nucleotide divergence } \\
\text { between species in low } \\
\text { recombination regions than } \\
\text { normal recombination }\end{array}$ & $\begin{array}{l}\text { Low recombination regions exhibit low nucleotide } \\
\text { diversity within species because of recurrent } \\
\text { hitchhiking or background selection }\end{array}$ & $\begin{array}{l}\text { Observe inflation in relative divergence measures } \\
\text { because diversity within species reduced }\end{array}$ \\
\hline $\begin{array}{l}\text { Species differences map more } \\
\text { strongly to regions of low } \\
\text { recombination than to other } \\
\text { regions }\end{array}$ & $\begin{array}{l}\text { Loci in regions of low recombination are easier to } \\
\text { map than loci elsewhere both because of } \\
\text { potentially greater combined effect of multiple loci } \\
\text { and because of stronger marker-QTL linkage }\end{array}$ & $\begin{array}{l}\text { Observe stronger associations of all trait } \\
\text { differences to markers in regions of low } \\
\text { recombination (relative to markers in regions } \\
\text { of high recombination) }\end{array}$ \\
\hline
\end{tabular}

Abbreviation: QTL, quantitative trait locus. 
et al., 2008) and house mice (Panithanarak et al., 2004). Although conceptually similar to the observations of high divergence in rearranged regions, this pattern is distinct because centromeric regions exhibit low recombination rates both within species and in species hybrids.

However, each of the empirical studies cited above specifically documented this pattern at least in part using relative divergence measures such as $\mathrm{F}_{\mathrm{ST}}$ and $\mathrm{Da}$ and interpreted in the context of regions of low recombination facilitating species divergence in the presence of gene flow. Regions of low recombination generally possess low nucleotide diversity within species (Nachman, 2002) resulting from recurrent hitchhiking (MaynardSmith and Haigh, 1974) or background selection (Charlesworth et al., 1993). In this context, Charlesworth (1998) elegantly described the problem of low nucleotide diversity increasing relative divergence measures, concluding that ' $F_{\mathrm{ST}}$ is strongly influenced by the level of within-population diversity [and] several published cases of differences in $F_{\mathrm{ST}}$ among regions of high and low recombination in Drosophila may be caused in this way.' Such regions would sustain an artificially high relative divergence even longer than the temporary artifact discussed above resulting from the spread of new chromosomal arrangements. Overall, higher relative divergence in regions of low recombination may be (1) artifactual, (2) exist even in species that do not hybridize and (3) not support a role of restricted recombination in allowing species to persist in the absence of other data (Table 1).

\section{Differentiating water from mirages}

\section{Divergence measures}

Our strongest recommendation is that researchers need to consider the inherent bias associated with using relative measures of divergence in testing the role of restricted recombination in maintaining species. As an illustration, we have compared Da (relative average divergence corrected for within species diversity: Nei, 1987) with Dxy (absolute average divergence) for the $M$ and $S$ races of Anopheles gambiae using the data from Stump et al. (2005) (Figure 1). Although a highly significant difference between races is apparent in Da, no significant difference is noted in Dxy. In fact, we observe a nonsignificant difference in the wrong direction: high recombination regions being more differentiated on average than low recombination regions. This result does not disprove the conclusions of the many studies of these races (Stump et al., 2005; Turner et al., 2005; Slotman et al., 2006), but it illustrates a problem of relative divergence measures. In this case, there is direct evidence of current hybridization between these races (Tripet et al., 2001), and recent introgression may have occurred.

Further, we emphasize that absolute measures of divergence are no panacea; relative measures were used in those studies specifically to factor out biases associated with within-race diversity. Using only absolute measures may be overly conservative because higher diversity within races in regions of high recombination may cause the appearance of higher divergence in such regions because of ancestral polymorphisms, consistent with the Anopheles data in Figure 1. When the two types

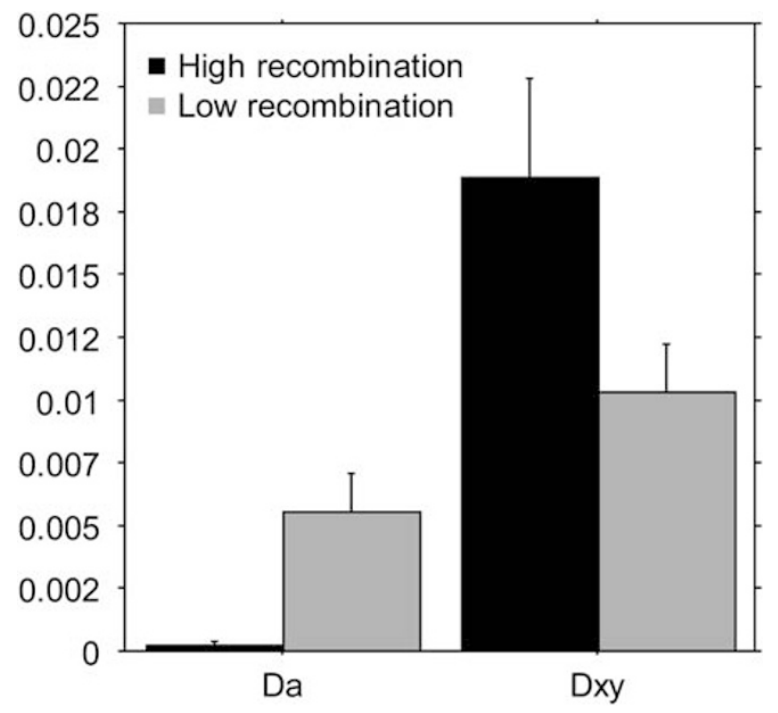

Figure $1 \mathrm{Da}$ and Dxy in low and high recombination regions between races of the $A$. gambiae X chromosome. The same loci are data points in both the Da and Dxy plots, and error bars indicate standard errors.

of measures give the same answer, one can have some confidence in the interpretation, but when they give different answers, then a bias is likely affecting one measure (either by overly deflating Da or by giving a high Dxy that does not reflect divergence occurring since the species split). The difficulty in the latter situation is interpreting which measure is biased or misinterpreted.

Mapping traits differentiating the species to such regions Models of restricted recombination maintaining species predict that trait differences between diverging races or species should map disproportionately to regions of low recombination. This pattern has been documented in the Drosophila pseudoobscura system (Noor et al., 2001b,c) and Helianthus sunflowers (Lai et al., 2005). Such mapping lends further support to studies showing higher DNA sequence differentiation in such regions. However, mapping studies can be biased by very similar phenomena: associations between markers and traits will, on average, be much stronger in regions of low recombination than regions of high recombination (Noor et al., 2001a: see Table 1; Feder and Nosil, 2009). This bias can be partially alleviated through higher marker density in regions of high recombination or if one finds that the low recombination regions alone contribute effects sufficient to explain the full interspecies difference.

Similarity in divergence across multiple rearrangements Species often differ by multiple, rather than single, rearrangements, and these systems offer a potential additional means for testing the importance of regions of restricted recombination. In such systems, one approach to differentiating ancient arrangements differentially segregating into diverging species (first problem in Table 1) from inversions arising after lineage split is to determine if absolute divergence (corrected for mutation rate) is similar across multiple arrangements, and consistently greater than in collinear regions (Kulathinal et al., 2009). Assuming the rearrangements arose at 
different times, consistent measures of divergence between species across all of them would suggest a model of species divergence in isolation with subsequent gene exchange and homogenization in collinear regions. However, this test assumes that the rearrangements arose at different times; if the rearrangements actually arose close in time to each other, then the test is uninformative. In addition, the test is conservative in that it assumes allopatric divergence and secondary contact-if the rearrangements were sequential and contributed to speciation in the face of gene flow, then they could exhibit quite different divergence times.

\section{Comparing co-occurring and allopatric populations}

Perhaps the most direct test for interspecies gene flow is to identify greater genetic similarity between species in populations that co-occur compared to allopatric populations, particularly in collinear regions of the genome. The restricted recombination model predicts that hybridizing species exchange genetic material in regions of normal recombination, but regions of low recombination remain differentiated because of their stronger associations with adaptive variants or barriers to gene flow such as hybrid sterility. Co-occurring populations receive this exchanged genetic material from the other species directly, and only later might foreign alleles spread to allopatric populations (for example, Nosil et al., 2003; Grant et al., 2005).

The difficulty with this test is that it requires that the populations within species exchange genetic material with each other at a rate comparable to or lower than interspecies gene flow. If intraspecies gene flow is high, then any genetic material obtained from other species will quickly spread to allopatric populations, and the 'signature' of introgression will not be detectable. As an illustration of this difficulty, Kulathinal and Singh (2000) found that Drosophila pseudoobscura populations co-occurring with vs allopatric to $D$. persimilis were similarly divergent from $D$. persimilis. However, a recent next-generation sequencing approach identified a slight, but marginally significant, difference in divergence from $D$. persimilis between co-occurring and allopatric D. pseudoobscura subspecies in collinear regions (Kulathinal et al., 2009), while inverted regions exhibited no difference in divergence, consistent with restricted recombination maintaining the co-occurring subspecies. Genetic mapping results demonstrating that hybrid sterility maps only to inverted regions in these co-occurring subspecies but to inverted and collinear regions in allopatric subspecies (Brown et al., 2004; Chang and Noor, 2007) further support this recent sequence data.

\section{More complex models}

The discussion above focused on simple approaches for detecting gene exchange between closely related species, as these have been used heavily in the context described. However, several models apply Markov chain Monte Carlo or other coalescent approaches to distinguish between shared variation through interspecies gene flow and ancestral polymorphism (Hey and Nielsen, 2004; Becquet and Przeworski, 2007). These models have also been used to infer gene exchange between species specifically in the context of the role of restricted recombination maintaining species. Although certainly more rigorous than the simple approaches described previously, these models also may bear assumptions not met in specific systems. A recent study showed that many realistic departures from the models' assumptions can lead to erroneous inference (Becquet and Przeworski, 2009). Tests inferring introgression through the length of contiguous introgressed DNA segments ('migrant tracts') may be used to alleviate this problem (Davison et al., 2009; Pool and Nielsen, 2009).

\section{Outlook}

Despite the bleak picture painted here, there are compelling reasons to expect that regions of restricted recombination (as by chromosomal rearrangements) can facilitate the formation or maintenance of good species, and diverse data support this contention. However, this model still requires careful evaluation, particularly in light of recent theoretical results that suggest differences in divergence between rearranged and collinear regions of hybridizing species may only persist a few thousand generations (Feder and Nosil, 2009), because differences in rearranged regions decay from rare gene conversion or double crossovers. This recent study provides an unusual situation where, at first glance, many results in nature do not appear consistent with theoretical predictions, suggesting that further work is needed to identify the sources of inconsistency.

Further, at some level, some of what we call 'biases' here about the restricted recombination model may be considered 'real': any genomic region that becomes 'isolated' by lack of recombination from alternate alleles in heterozygotes is effectively a 'genotypic cluster' as considered in some species concepts (Mallet, 1995). This would be true for the first bias listed in Table 1: all individuals carrying a new arrangement are recombinationally isolated from individuals carrying progenitor arrangement in that region. However, in practice, no one would argue that every new chromosomal rearrangement that restricts recombination from its progenitor should form an entity that should have its bearer dubbed a new species.

That said, inferring the role of restricted recombination in species persistence for a particular system warrants extra caution, particularly given that intraspecific processes create a signature similar to one predicted by this model (Table 1). Our intention here is not to attack particular proposed cases or studies but instead to draw attention to this concern for future work and inferences. Indeed, many of the studies cited have applied multiple lines of evidence, rather than a single line, to test the hypothesis that regions of restricted recombination fail to cross species boundaries. Critical to testing this hypothesis is unambiguously identifying both that interspecies gene flow has occurred and that it happens disproportionately in regions of higher recombination. We urge caution in future studies and awareness of the likely biases, hence reducing the possibility that we will be misled by 'mirages' while seeking water.

\section{Conflict of interest}

The authors declare no conflict of interest. 


\section{Acknowledgements}

We thank S McGaugh, P Nosil, L Stevison and an anonymous referee for helpful comments. The authors are supported by funding from the National Science Foundation and National Institutes of Health.

\section{References}

Ayala FJ, Coluzzi M (2005). Chromosome speciation: humans, Drosophila, and mosquitoes. Proc Natl Acad Sci USA 102(Suppl 1): 6535-6542.

Basset P, Yannic G, Brunner H, Hausser J (2006). Restricted gene flow at specific parts of the shrew genome in chromosomal hybrid zones. Evolution 60: 1718-1730.

Basset P, Yannic G, Hausser J (2008). Chromosomal rearrangements and genetic structure at different evolutionary levels of the Sorex araneus group. J Evol Biol 21: 842-852.

Beaumont MA (2005). Adaptation and speciation: what can F(st) tell us? Trends Ecol Evol 20: 435-440.

Becquet C, Przeworski M (2007). A new approach to estimate parameters of speciation models with application to apes. Genome Res 17: 1505-1519.

Becquet C, Przeworski M (2009). Learning about modes of speciation by computational approaches. Evolution (in press).

Brown KM, Burk LM, Henagan LM, Noor MAF (2004). A test of the chromosomal rearrangement model of speciation in Drosophila pseudoobscura. Evolution 58: 1856-1860.

Butlin RK (2005). Recombination and speciation. Mol Ecol 14: 2621-2635.

Chang AS, Noor MAF (2007). The genetics of hybrid male sterility between the allopatric species pair Drosophila persimilis and D. pseudoobscura bogotana: dominant sterility alleles in collinear autosomal regions. Genetics $\mathbf{1 7 6}$ 343-349.

Charlesworth B (1998). Measures of divergence between populations and the effect of forces that reduce variability. Mol Biol Evol 15: 538-543.

Charlesworth B, Morgan MT, Charlesworth D (1993). The effect of deleterious mutations on neutral molecular variation. Genetics 134: 1289-1303.

Davison D, Pritchard JK, Coop G (2009). An approximate likelihood for genetic data under a model with recombination and population splitting. Theor Popul Biol 75: 331-345.

Feder JL, Nosil P (2009). Chromosomal inversions and species differences: when are genes affecting adaptive divergence and reproductive isolation expected to reside within inversions? Evolution (in press)

Feder JL, Roethele JB, Filchak K, Niedbalski J, Romero-Severson J (2003). Evidence of inversion polymorphism related to sympatric host race formation in the apple maggot fly, Rhagoletis pomonella. Genetics 163: 939-953.

Geraldes A, Basset P, Gibson B, Smith KL, Harr B, Yu H-T et al. (2008). Inferring the history of speciation in house mice from autosomal, X-linked, Y-linked and mitochondrial genes. Mol Ecol 17: 5349-5363.

Grant PR, Grant BR, Petren K (2005). Hybridization in the recent past. Am Nat 166: 56-67.

Hey J (2006). Recent advances in assessing gene flow between diverging populations and species. Curr Opin Genet Dev 16: 592-596.

Hey J, Nielsen R (2004). Multilocus methods for estimating population sizes, migration rates and divergence time, with applications to the divergence of Drosophila pseudoobscura and D. persimilis. Genetics 167: 747-760.

Hoffmann AA, Rieseberg LH (2008). Revisiting the impact of inversions in evolution: from population genetic markers to drivers of adaptive shifts and speciation? Ann Rev Evol Evol Syst 39: 21-42.
Joly S, McLenachan PA, Lockhart PJ (2009). A statistical approach for distinguishing hybridization and incomplete lineage sorting. Am Nat 174: E54-E70.

Kandul NP, Lukhtanov VA, Pierce NE (2007). Karyotypic diversity and speciation in Agrodiaetus butterflies. Evolution 61: 546-559.

Kirkpatrick M, Barton N (2006). Chromosome inversions, local adaptation and speciation. Genetics 173: 419-434.

Kulathinal RJ, Singh RS (2000). A biogeographic approach for testing the role of reinforcement: the case of Drosophila pseudoobscura and D. persimilis. Evolution 54: 210-217.

Kulathinal RJ, Stevison LS, Noor MAF (2009). The genomics of speciation in Drosophila: diversity, divergence, and introgression estimated using low-coverage genome sequencing. PLoS Genet 5: e1000550.

Lai Z, Nakazato T, Salmaso M, Burke JM, Tang S, Knapp SJ et al. (2005). Extensive chromosomal repatterning and the evolution of sterility barriers in hybrid sunflower species. Genetics 171: 291-303.

Lewontin RC, Moore JA, Provine WB, Wallace B (eds). (1981). Dobzhansky's Genetics of Natural Populations I-XLIII. Columbia University Press: New York.

Llopart A, Lachaise D, Coyne JA (2005). An anomalous hybrid zone in Drosophila. Evolution 59: 2602-2607.

Machado CA, Haselkorn TS, Noor MAF (2007a). Evaluation of the genomic extent of effects of fixed inversion differences on intraspecific variation and interspecific gene flow in Drosophila pseudoobscura and D. persimilis. Genetics 175: 1289-1306.

Machado CA, Kliman RM, Markert JA, Hey J (2002). Inferring the history of speciation from multilocus sequence data: the case of Drosophila pseudoobscura and its close relatives. Mol Biol Evol 19: 472-488.

Machado CA, Matzkin LM, Reed LK, Markow TA (2007b). Multilocus nuclear sequences reveal intra- and interspecific relationships among chromosomally polymorphic species of cactophilic Drosophila. Mol Ecol 16: 3009-3024.

Mallet J (1995). A species definition for the Modern Synthesis. Trends Ecol Evol 10: 294-299.

MaynardSmith J, Haigh J (1974). The hitch-hiking effect of a favourable gene. Genet Res 23: 23-35.

Michel AP, Grushko O, Guelbeogo WM, Lobo NF, Sagnon N, Costantini C et al. (2006). Divergence with gene flow in Anopheles funestus from the Sudan Savanna of Burkina Faso, West Africa. Genetics 173: 1389-1395.

Nachman MW (2002). Variation in recombination rate across the genome: evidence and implications. Curr Opin Genet Dev 12: 657-663.

Navarro A, Barton NH (2003). Accumulating postzygotic isolation in parapatry: a new twist on chromosomal speciation. Evolution 57: 447-459.

Nei M (1987). Molecular Evolutionary Genetics. Columbia University Press: New York.

Noor MA, Garfield DA, Schaeffer SW, Machado CA (2007). Divergence between the Drosophila pseudoobscura and D. persimilis genome sequences in relation to chromosomal inversions. Genetics 177: 1417-1428.

Noor MAF, Cunningham AL, Larkin JC (2001a). Consequences of recombination rate variation on quantitative trait locus mapping studies: simulations based on the Drosophila melanogaster genome. Genetics 159: 581-588.

Noor MAF, Grams KL, Bertucci LA, Almendarez Y, Reiland J, Smith KR (2001b). The genetics of reproductive isolation and the potential for gene exchange between Drosophila pseudoobscura and $D$. persimilis via backcross hybrid males. Evolution 55: 512-521.

Noor MAF, Grams KL, Bertucci LA, Reiland J (2001c). Chromosomal inversions and the reproductive isolation of species. Proc Natl Acad Sci USA 98: 12084-12088.

Nosil P, Crespi BJ, Sandoval CP (2003). Reproductive isolation driven by the combined effects of ecological adaptation and reinforcement. Proc R Soc London B 270: 1911-1918. 
Nosil P, Funk DJ, Ortiz-Barrientos D (2009). Divergent selection and heterogeneous genomic divergence. Mol Ecol 18: 375-402.

Panithanarak T, Hauffe HC, Dallas JF, Glover A, Ward RG, Searle JB (2004). Linkage-dependent gene flow in a house mouse chromosomal hybrid zone. Evolution 58: 184-192.

Pool JE, Nielsen R (2009). Inference of historical changes in migration rate from the lengths of migrant tracts. Genetics 181: 711-719.

Powell JR, Petrarca V, della Torre A, Caccone A, Coluzzi M (1999). Population structure, speciation, and introgression in the Anopheles gambiae complex. Parassitologia 41: 101-113.

Rieseberg LH (2001). Chromosomal rearrangements and speciation. Trends Ecol Evol 16: 351-358.

Rieseberg LH, Whitton J, Gardner K (1999). Hybrid zones and the genetic architecture of a barrier to gene flow between two sunflower species. Genetics 152: 713-727.

Singh BN (2001). Patterns of inversion polymorphism in three species of the Drosophila melanogaster species group. Indian J Exp Biol 39: 611-622.

Slotman MA, Reimer LJ, Thiemann T, Dolo G, Fondjo E, Lanzaro GC (2006). Reduced recombination rate and genetic differentiation between the $\mathrm{M}$ and $\mathrm{S}$ forms of Anopheles gambiae s.s. Genetics 174: 2081-2093.
Stevison LS (2008). Hybridization and gene flow. Nat Educ 1: 1. Strasburg JL, Scotti-Saintagne C, Scotti I, Lai Z, Rieseberg LH (2009). Genomic patterns of adaptive divergence between chromosomally differentiated sunflower species. Mol Biol Evol 26: 1341-1355.

Stump AD, Fitzpatrick MC, Lobo NF, Traore S, Sagnon N, Costantini C et al. (2005). Centromere-proximal differentiation and speciation in Anopheles gambiae. Proc Natl Acad Sci USA 102: 15930-15935.

Tripet F, Toure YT, Taylor CE, Norris DE, Dolo G, Lanzaro GC (2001). DNA analysis of transferred sperm reveals significant levels of gene flow between molecular forms of Anopheles gambiae. Mol Ecol 10: 1725-1732.

Turner TL, Hahn MW, Nuzhdin SV (2005). Genomic islands of speciation in Anopheles gambiae. PLoS Biol 3: e285.

Wakeley J, Hey J (1997). Estimating ancestral population parameters. Genetics 145: 847-855.

Yannic G, Basset P, Hausser J (2009). Chromosomal rearrangements and gene flow over time in an inter-specific hybrid zone of the Sorex araneus group. Heredity 102: 616-625.

Yatabe Y, Kane NC, Scotti-Saintagne C, Rieseberg LH (2007). Rampant gene exchange across a strong reproductive barrier between the annual sunflowers, Helianthus annuus and H. petiolaris. Genetics 175: 1883-1893. 